\title{
STRONG CONVERGENCE OF FUNCTIONS ON KÖTHE SPACES
}

\author{
BY \\ GERALD SILVERMAN
}

\begin{abstract}
Let $\Lambda$ be a rearrangement invariant Köthe space over a nondiscrete group $G$ with Haar measure $\mu$. For a function $f \in \Lambda$ and relatively compact 0 -neighborhood $U$ in $G$ the function$$
T_{U} f(x)=\frac{1}{\mu(U)} \cdot \int_{U+x} f d \mu
$$

is continuous and also belongs to $\Lambda$. The convergence $T_{U} f \rightarrow f$ (as $U \rightarrow 0$ ) for the strong Köthe topology on $\Lambda$ is involved in establishing compactness criteria for subsets of a Köthe space. The main result of this paper is a necessary and sufficient condition for convergence $T_{U} f \rightarrow f$ in the strong topology on $\Lambda$.
\end{abstract}

1. In [3], [4] and [5] Köthe studied pairs of subspaces of real sequences that were in weak duality. Dieudonné later generalized the theory to subspaces of locally integrable functions over a locally compact measure space $E$ with Radon measure $\mu$. If $E$ is a $\sigma$-compact, locally compact Hausdorff space with regular Radon measure $\mu$, we let $\Omega$ be the space of all functions which are integrable on each compact set in $E$. For a subset $\Gamma$ of $\Omega$, the Köthe space associated with $\Gamma$ is $\Lambda=\Lambda(\Gamma)=\left\{f \in \Omega: \int_{E}|f g| d \mu<\infty\right.$ for all $\left.g \in \Gamma\right\}$ and the Köthe dual is $\Lambda^{*}=\Lambda^{*}(\Gamma)$ $=\Lambda(\Lambda(\Gamma))$. The pair $\left(\Lambda, \Lambda^{*}\right)$ is in weak duality; an example of such a pair is $\left(L^{p}, L^{q}\right)$. The set $\Lambda$ can be made into a complete locally convex topological vector space under the strong topology $S\left(\Lambda, \Lambda^{*}\right)=S$ defined by the seminorms

$$
S_{H}(f)=\sup _{g \in H} \int|f g| d \mu
$$

as $H$ runs through the weakly bounded subsets of the Köthe dual $\Lambda^{*}$.

For $E=G$ an additive topological group, $f \in \Omega$, and $U$ a relatively compact 0 -neighborhood in $G$, we define the continuous function

$$
T_{U} f(x)=\frac{1}{\mu(U)} \cdot \int_{U+x} f d \mu .
$$

In [2] these functions were used in giving compactness criteria for subsets of Köthe spaces over $G$. The importance of the convergence of $T_{U} f$ to $f$ (for the strong

Received by the editors July 8, 1970.

AMS 1969 subject classifications. Primary 4635.

Key words and phrases. Köthe spaces, monotonic rearrangements, equimeasurability, nonatomic measure, strong Köthe topology.

Copyright (C) 1972, American Mathematical Society 
topology) as $U$ runs through the relatively compact neighborhoods of 0 in $G$ for each function $f$ in a Köthe space will appear in papers by Welland and Goes which are as yet unpublished. In this paper a necessary and sufficient condition is established for the convergence of $T_{U} f$ to $f$ for the strong topology on a rearrangement invariant Köthe space.

2. Two functions $f$ and $g$ are said to be equimeasurable or rearrangement invariant if

$$
\mu(\{x:|f(x)|>r\})=\mu(\{x:|g(x)|>r\})
$$

for all nonnegative $r$. A Köthe space $\Lambda$ is rearrangement invariant if $f^{\prime} \in \Lambda$ whenever $f^{\prime}$ is equimeasurable with some $f \in \Lambda$. It is known that such a $\Lambda$ is contained in the direct sum of $L^{1}(E, \mu)$ and $L^{\infty}(E, \mu)$, and that the Köthe dual $\Lambda^{*}$ is also rearrangement invariant (proved in [7]).

A set of functions $H$ is normal if $g \in H$ and $|h| \leqq|g|$ implies $h \in H$. In [6] it was proven that if $E$ has no atoms (i.e. a set $S \subset E$ of positive measure such that $S_{1} \subset S$ implies $\mu\left(S_{1}\right)=0$ or $\left.\mu\left(S_{1}\right)=\mu(S)\right)$ there is a fundamental system of normal, rearrangement invariant, weakly bounded subsets $\{H\}$ of $\Lambda^{*}$ for which the seminorms $S_{H}$, which generate the strong topology of $\Lambda$, have the property that $S_{H}(f)$ $=S_{H}\left(f^{\prime}\right)$ for $f$ equimeasurable with $f^{\prime}$.

In this paper $G$ will be a $\sigma$-compact, locally compact, Hausdorff, nondiscrete topological group; $\mu$ will denote invariant Haar measure on $G$. The family of relatively compact neighborhoods of 0 in $G$ will be denoted by $\mathscr{U}$. In addition, we will often write $\int_{G} f(x) d x$ to mean $\int_{G} f d \mu$, and $f_{y}$ to be the function $f_{y}(x)=f(y+x)$ for $f \in \Omega$ and $y \in G$.

3. The following lemma will enable us to use the information we know about rearrangement invariant Köthe spaces over a nonatomic space $G$.

LEMMA 1. If $G$ does not have the discrete topology, then Haar measure $\mu$ is nonatomic; that is $G$ has no atoms.

Proof. We first show that if $G$ contains an atom $S$, then $\mu(S)$ must be finite. If not, and $\left\{K_{n}\right\}_{n=1}^{\infty}$ is the increasing sequence of compact sets whose union is $G$, then $\mu\left(K_{n} \cap S\right)$ is finite $(n=1,2, \ldots)$ and strictly less than $\mu(S)=\infty$. It follows that $\mu\left(K_{n} \cap S\right)=0$ for each $n$, and $\mu(S)=0$; this is a contradiction. Therefore we must assume that $\mu(S)<\infty$. Now since $\mu(S)=\sup \{\mu(K): K \subset S, K$ is compact $\}$ and $S$ is an atom, there is a compact set $K$ which is an atom satisfying $\mu(K)=\mu(S)$. By the nondiscreteness of $G$, there is a nonempty open set $U$ containing 0 such that $\mu(U)<\mu(K)$. Since $\{U+x: x \in K\}$ is an open cover of the compact $K$, there must be a finite number of elements $\left\{x_{1}, x_{2}, \ldots, x_{n}\right\}$ in $K$ such that $\bigcup_{i=1}^{n} U+x_{i} \supset K$. 
But $\mu\left(U+x_{i} \cap K\right) \leqq \mu(U)<\mu(K)(i=1,2, \ldots, n)$ implies $\mu\left(U+x_{i} \cap K\right)=0$. It then follows that $\mu(S)=\mu(K)=0$.

LEMMA 2. If $\Lambda$ is a rearrangement invariant Köthe space over $G, U$ is a compact neighborhood of 0 in $G$ and $f$ is a function in $\Lambda$, then

(i) $T_{U} f \in \Lambda$;

(ii) $T_{U} f$ is a uniformly continuous function on $G$;

(iii) $\rho\left(T_{U} f\right) \leqq \rho(f)$ as $\rho$ runs through a certain family of seminorms that generate the strong topology of $\Lambda$.

Proof. If $f \in \Lambda$ and $U$ is compact in $G$, we show that $T_{U} f \cdot g$ is integrable for every $g \in \Lambda^{*}$; that is, $T_{U} f \in \Lambda$. As $\Lambda^{*}$ is rearrangement invariant, there is a normal, rearrangement invariant, weakly bounded subset $H$ of $\Lambda^{*}$ which $g$ belongs to, and satisfying $S_{H}\left(f^{\prime}\right)=S_{H}(f)$ whenever $f^{\prime}$ is equimeasurable with $f$. Since $f_{y}$ is equimeasurable with $f$ for each $y \in G$, we have

$$
\begin{aligned}
\int_{G}\left|T_{U} f(x) \cdot g(x)\right| d x & =\int_{G} \frac{1}{\mu(U)} \cdot\left|\int_{U} f(x+y) \cdot g(x) \cdot d y\right| \cdot d x \\
& \leqq \frac{1}{\mu(U)} \cdot \int_{G} \int_{U}|f(x+y) \cdot g(x)| \cdot d y \cdot d x \\
& =\frac{1}{\mu(U)} \cdot \int_{U} \int_{G}|f(x+y) \cdot g(x)| \cdot d x \cdot d y \\
& \leqq \frac{1}{\mu(U)} \cdot \int_{U} \sup _{h \in H} \int|f(x+y) \cdot h(x)| d x \cdot d y \\
& =\frac{1}{\mu(U)} \int_{U} S_{H}\left(f_{y}\right) d y \\
& =\frac{1}{\mu(U)} \int_{U} S_{H}(f) d \mu=S_{H}(f)<\infty
\end{aligned}
$$

Thus $T_{U} f \in \Lambda$. Furthermore, it is clear that $\int_{G} T_{U} f \cdot g \cdot d \mu \leqq S_{H}(f)$ for all functions $g \in H$. Taking the supremum on all $g \in H$, we obtain $S_{H}\left(T_{U} f\right) \leqq S_{H}(f)$. Since the seminorms $S_{H}$ generate the strong topology of $\Lambda$, (iii) is proved. In addition, this also shows that $T_{U}: \Lambda \rightarrow \Lambda$ is a strongly continuous linear function.

In order to show that $T_{U} f$ is uniformly continuous for $U$ compact and $f \in \Lambda$, we observe first that $f=h+g$ where $h \in L^{1}(G, \mu)$ and $g \in L^{\infty}(G, \mu)$ (since $\Lambda$ is rearrangement invariant); we then have $T_{U} f=T_{U} h+T_{U} g$. We must show that for any $\varepsilon>0$ there is a 0 -neighborhood $V$ in $G$ such that $x-y \in V$ implies $\left|T_{U} f(x)-T_{U} f(y)\right|<\varepsilon$.

Let $\varepsilon>0$ be given. Since $h$ is integrable, there is a $\delta>0$ such that $A$ measurable and $\mu(A)<\delta$ implies $\int_{A}|h| d \mu<\varepsilon / 4$. By the compactness of $U$ in $G$ and the regularity of $\mu$, there is a symmetrical 0-neighborhood $V$ such that $\mu(V+U \backslash U)$ 
$<\min \left\{\frac{1}{4} \cdot \varepsilon /\|g\|_{\infty} ; \delta\right\}$. Then for $x-y \in V$, we will have

$$
\begin{aligned}
\mu(U) & \cdot\left|T_{U} f(x)-T_{U} f(y)\right| \\
= & \mu(U) \cdot\left|T_{U} h(x)-T_{U} h(y)+T_{U} g(x)-T_{U} g(y)\right| \\
\leqq & \left|\int_{U+x} h(t) d t-\int_{U+y} h(t) \cdot d t\right|+\left|\int_{U+x} g(t) d t-\int_{U+y} g(t) d t\right| \\
\leqq & \int_{x+U \backslash y+U}|h(t)| d t+\int_{y+U \backslash x+U}|h(t)| d t+\int_{x+U \backslash y+U}|g(t)| d t+\int_{y+U \mid x+U}|g(t)| d t \\
= & \int_{x-y+U \backslash U}|h(t+y)| \cdot d t+\int_{y-x+U \backslash U}|h(t+x)| d t+\int_{x-y+U \backslash U}|g(t+y)| \cdot d t \\
& +\int_{y-x+U \backslash U}|g(t+x)| d t \\
\leqq & \int_{V+U \backslash U}|h(t+y)| d t+\int_{V+U \backslash U}|h(t+x)| d t+\int_{V+U \backslash U}|g(t+y)| d t \\
& +\int_{V+U \backslash U}|g(t+x)| d t \\
< & \frac{\varepsilon}{4}+\frac{\varepsilon}{4}+2 \cdot\|g\|_{\infty} \cdot \frac{\varepsilon}{\|g\|_{\infty}} \cdot \frac{1}{4}=\varepsilon .
\end{aligned}
$$

Thus $T_{U} f$ is uniformly continuous.

REMARK. On the real line such functions $T_{U} f$ are of the form

$$
T^{h} f(x)=\frac{1}{h} \int_{x-h / 2}^{x+h / 2} f(t) d t \text { for } h>0 .
$$

The $T^{h}$ operation takes a function $f$ and smoothes it out to a function that approximates the original function; in fact, $\lim _{h \rightarrow \infty} T^{h} f(x)=f(x)$ a.e. whenever $f$ is locally integrable. As an example let us consider $f=\chi_{[a, b]}$ where $a$ and $b$ are real numbers with $a<b$. Then

$$
\begin{aligned}
T^{h} f(x) & =(x-(a-h / 2)) / h & & \text { for } a-h / 2 \leqq x \leqq a+h / 2 ; \\
& =1 & & \text { for } a+h / 2 \leqq x \leqq b-h / 2 \\
& =(b+h / 2-x) / h & & \text { for } b-h / 2 \leqq x \leqq b+h / 2
\end{aligned}
$$

Obviously, $\lim _{h \rightarrow 0} T^{h} f(x)=1$ for $x \in(a, b)$.

We now give an example of two locally integrable functions $f$ and $g$ on $R^{1}$ such that $\int_{R^{1}} f(t) \cdot g(t)=0$, but $\int_{R^{1}} T^{h} f(t) \cdot g(t) d t=\infty$ for all $h>0$. This example will show that there is a Köthe space over $R^{1}, \Lambda=L_{g}^{1}=\left\{f \in \Omega: \int_{R^{1}}|f g| d \mu<\infty\right\}$, such that $f \in \Lambda$, but $T^{h} f \notin \Lambda$ for any $h$.

For each integer $n \geqq 5$ we choose numbers $a_{n}, b_{n}, c_{n}$ and $d_{n}$ such that $n<a_{n}<b_{n}$ $<c_{n}<d_{n}, b_{n}-a_{n}=c_{n}-b_{n}=d_{n}-c_{n}=1 / n$, and $n+1-d_{n}=a_{n}-n<\frac{1}{8}$. Set $A_{n}=\left(a_{n}, b_{n}\right)$ $\cup\left(c_{n}, d_{n}\right), \quad B_{n}=\left(b_{n}, c_{n}\right) \quad(n \geqq 5), f=\sum_{n=5}^{\infty} n \cdot \chi_{A_{n}}$ and $g=\sum_{n=5}^{\infty} n \cdot \chi_{B_{n}}$. Clearly, 
$\int_{R^{1}} f(t) \cdot g(t) d t=0$. However, choosing $h \leqq \frac{1}{8}$, we obtain

$$
\begin{aligned}
\int_{-\infty}^{\infty} T^{h} f(x) \cdot g(x) d x & =\int_{-\infty}^{\infty} \frac{1}{h} \cdot \int_{-h / 2}^{h / 2} f(x+t) \cdot g(x) d t d x \\
& =\frac{1}{h} \cdot \int_{-h / 2}^{h / 2} \int_{-\infty}^{\infty} f(x+t) \cdot g(x) d x d t \\
& =\frac{1}{h} \cdot \int_{-h / 2}^{h / 2} \sum_{n=5}^{\infty} \int_{n}^{n+1} n^{2} \cdot \chi_{A_{n}}(x+t) \cdot \chi_{B_{n}}(x) d x d t \\
& =\sum_{n=5}^{\infty} \frac{1}{h} \cdot \int_{-h / 2}^{h / 2} n^{2} \cdot \mu\left(A_{n}-t \cap B_{n}\right) d t \\
& \geqq \frac{1}{h} \sum_{n \geq 2 / h} n^{2} \cdot \int_{0}^{1 / n} \mu\left(A_{n}+t \cap B_{n}\right) d t \\
& =\frac{1}{h} \sum_{n \geqq 2 / h} n^{2} \cdot \int_{0}^{1 / n} t \cdot d t=\frac{1}{h} \cdot \sum_{n \geqq 2 / h} n^{2} \cdot \frac{1}{n^{2}} \cdot \frac{1}{2}=\infty .
\end{aligned}
$$

In the following theorem $C_{0}=C_{0}(G)$ will denote the continuous functions of compact support on $G$ and $S\left(\Lambda, \Lambda^{*}\right)=S$ will denote the strong Köthe topology on $\Lambda$ generated by the rearrangement invariant seminorms

$$
S_{B}(f)=\sup _{g \in B} \int_{G} f g d \mu \quad(\text { for } f \in \Lambda)
$$

where $B$ runs through the normal, rearrangement invariant, weakly bounded subsets of $\Lambda^{*}$. Recall that $\lim _{U \in \mathscr{U}} T_{U} f=f$ for the topology $S$ means that for each weakly bounded $B \subset \Lambda^{*}$ there is a relatively compact 0-neighborhood $U_{0}$ in $G$ such that $S_{B}\left(T_{U} f-f\right) \leqq 1$ for all 0 -neighborhoods $U \subset U_{0}$.

THEOREM. If $\Lambda$ is a rearrangement invariant Köthe space over $G$ with the strong topology $S$, then the following are equivalent:

(1) $C_{0}$ is dense in $\Lambda$;

(2) $\lim _{U \in \mathscr{U}} T_{U} f=$ f for each $f \in \Lambda ; \lim _{n \rightarrow \infty} \varphi \cdot \chi_{K_{n}}=\varphi$ for each nonnegative uniformly continuous $\varphi$ in $\Lambda$.

Proof. The implication (1) implies (2) will be proven first. We begin by showing that $\lim _{U \in \mathscr{U}} T_{U} \varphi=\varphi$ for each $\varphi \in C_{0}$. Let $\varphi \in C_{0}$ have its support on $K$ compact, and let $B$ be a normal, weakly bounded subset of $\Lambda^{*}$. If $U$ is a compact and symmetric 0-neighborhood in $G$, then $U+K=\{u+k: u \in U, k \in K\}$ is compact, $d=S_{B}\left(\chi_{U+K}\right)$ is a finite number, and the support of $T_{U} \varphi$ is contained in $U+K$. In order to see this last statement we observe that for $x \in E \backslash U+K$ we will have $U+x \cap K=\varnothing$; for if there was a $u \in U$ and $k \in K$ satisfying $u+x=k$, we would have $x=k-u \in K+U$ (as $U$ is symmetrical), a contradiction. Thus for $x \in E \backslash U+K$, we obtain

$$
T_{U} \varphi(x)=\frac{1}{\mu(U)} \cdot \int_{U+x} \varphi(y) d y=\frac{1}{\mu(U)} \cdot \int_{U+x \cap K} \varphi(y) d y=0 .
$$


If $d=0$ the above argument shows that $S_{B}\left(T_{V} \varphi-\varphi\right)=0$ for all 0-neighborhoods $V \subset U$. We can then assume that $d>0$. Now since $\varphi$ is uniformly continuous, there is a symmetric 0-neighborhood $V$ with $V+V \subset U$ such that $x-y \in V$ implies $|\varphi(x)-\varphi(y)|<1 / d$. The 0 -neighborhood $V$ is relatively compact, and for any 0 -neighborhood $V^{\prime} \subset V$ we have

$$
\begin{aligned}
S_{B}\left(T_{V^{\prime}} \varphi-\varphi\right) & =S_{B}\left(\chi_{K+U} \cdot\left(T_{V^{\prime}} \varphi-\varphi\right)\right) \\
& =\sup _{g \in B} \int_{G}\left|\chi_{K+U}(x) \cdot\left(T_{V^{\prime}} \varphi(x)-\varphi(x)\right) \cdot g(x)\right| d x \\
& \leqq \sup _{g \in B} \int_{G} \chi_{K+U}(x) \cdot\left\{\frac{1}{\mu\left(V^{\prime}\right)} \cdot \int_{V^{\prime}}|\varphi(x)-\varphi(x+y)| d y\right\} \cdot g(x) d x \\
& <\sup _{g \in B} \int_{G} \chi_{K+U} \cdot \frac{1}{d} \cdot g \cdot d \mu=\frac{1}{d} \cdot S_{B}\left(\chi_{K+U}\right)=1 .
\end{aligned}
$$

Thus $\lim _{U \in \mathscr{U}} T_{U} \varphi=\varphi$ for each $\varphi \in C_{0}$. Fix $f \in \Lambda$. We now show $T_{U} f \rightarrow f$ in $\Lambda$. Let $B$ be a normal, rearrangement invariant, weakly bounded subset of $\Lambda^{*}$ whose associated seminorm $S_{B}$ is rearrangement invariant. Since $C_{0}$ is strongly dense in $\Lambda$, there is a $\varphi \in C_{0}$ such that $S_{B}(\varphi-f)<\frac{1}{3}$.

The convergence of $T_{U} \varphi$ to $\varphi$ implies there is a relatively compact 0 -neighborhood $U_{0}$ such that $S_{B}\left(T_{U} \varphi-\varphi\right)<\frac{1}{3}$ for all 0-neighborhoods $U \subset U_{0}$. By Lemma 2 we have $S_{B}\left(T_{U} f-f\right) \leqq S_{B}\left(T_{U} f-T_{U} \varphi\right)+S_{B}\left(T_{U} \varphi-\varphi\right)+S_{B}(\varphi-f) \leqq 2 \cdot S_{B}(f-\varphi)+S_{B}\left(T_{U} \varphi-\varphi\right)<1$ for $U \subset U_{0}$. Thus $\lim _{U \in \mathscr{U}} T_{U} f=f$ for each $f \in \Lambda$.

For the second part of (1) implies (2) we suppose that $\psi \in \Lambda$ is a nonnegative and uniformly continuous function. If $B \subset \Lambda^{*}$ is weakly bounded and normal, there is a function $\varphi \in C_{0}$ such that $S_{B}(\psi-\varphi)<1$. Suppose the support of $\varphi$ is contained in $K_{m}$ for some integer $m$. If $n \geqq m$ we have

$$
\begin{gathered}
0=\left|\psi(x)-\chi_{K_{n}} \cdot \psi(x)\right| \leqq|\varphi(x)-\psi(x)| \text { for any } x \in K_{n} \\
\left|\psi(x)-\chi_{K_{n}} \cdot \psi(x)\right|=|\psi(x)|=|\psi(x)-\varphi(x)| \text { for } x \in E \mid K_{n} .
\end{gathered}
$$

Therefore, for $n \geqq m$ we have

which was to be shown.

$$
S_{B}\left(\psi-\chi_{K_{n}} \cdot \psi\right) \leqq S_{B}(\psi-\varphi)<1,
$$

In order to prove (2) implies (1) we must first prove that if $B$ is a normal, rearrangement invariant, weakly bounded subset of $\Lambda^{*}$ whose associated seminorm $S_{B}$ is rearrangement invariant, and $\varepsilon>0$ is arbitrary, then there is a $\delta>0$ (dependent upon $\varepsilon$ ) such that $S_{B}\left(\chi_{A}\right)<\varepsilon$ for any measurable set $A$ satisfying $\mu(A)<\delta$. Since $G$ is nondiscrete, there is a compact set $K$ with nonempty interior $K^{\circ}$ for which the boundary of $K, \partial K=K \mid K^{\circ}$, contains a point $x_{0}$ with the property that every open set about $x_{0}$ has a nonempty intersection with the open sets $K^{\circ}$ and $E \backslash K$. Since we have assumed $\lim _{U \in \mathscr{U}} T_{U} \chi_{K}=\chi_{K}$ for the strong topology of $\Lambda$, there is a compact, symmetric 0-neighborhood $U_{0}$ in $G$ such that $S_{B}\left(T_{U} \chi_{K}-\chi_{K}\right)<\varepsilon / 2$ for any 0 neighborhood $U \subset U_{0}$. Now we show that there is a 0 -neighborhood $V_{0} \subset U_{0}$ such 
that $T_{V_{0}} \chi_{K}(x)>\frac{1}{2}$ for all $x$ in some nonempty open set contained in $E \backslash K$. As $U_{0}+x_{0}$ is an open neighborhood of $x_{0}$ meeting $K^{\circ}$ in a nonempty open set and $\mu$ is a regular measure, there is a symmetric 0-neighborhood $U^{\prime} \subset U_{0}$ such that $0<\mu\left(U^{\prime}\right)$ $<\mu\left(U_{0}+x_{0} \cap K\right)$. The 0-neighborhood $V_{0}=\left(U^{\prime}+x_{0} \cap E \backslash K\right) \cup\left(U_{0}+x_{0} \cap K\right)-x_{0}$ $\supset\left(U^{\prime}+x_{0} \cap E \backslash K\right) \cup\left(U^{\prime}+x_{0} \cap K\right)-x_{0}=U^{\prime}$ is contained in $U_{0}$.

Since $\mu\left(U^{\prime}+x_{0} \cap E \backslash K\right) \leqq \mu\left(U^{\prime}+x_{0}\right)=\mu\left(U^{\prime}\right)<\mu\left(U_{0}+x_{0} \cap K\right)$, we have

$$
\begin{aligned}
T_{V_{0} \chi_{K}}\left(x_{0}\right) & =\frac{1}{\mu\left(V_{0}\right)} \cdot \int_{V_{0}+x_{0}} \chi_{K} d \mu=\frac{\mu\left(V_{0}+x_{0} \cap K\right)}{\mu\left(V_{0}+x_{0}\right)} \\
& =\frac{\mu\left(V_{0}+x_{0} \cap K\right)}{\mu\left(U^{\prime}+x_{0} \cap E \backslash K\right)+\mu\left(U_{0}+x_{0} \cap K\right)} \\
& =\frac{\mu\left(U_{0}+x_{0} \cap K\right)}{\mu\left(U^{\prime}+x_{0} \cap E \backslash K\right)+\mu\left(U_{0}+x_{0} \cap K\right)} \\
& >\frac{\mu\left(U_{0}+x_{0} \cap K\right)}{\mu\left(U_{0}+x_{0} \cap K\right)+\mu\left(U_{0}+x_{0} \cap K\right)}=\frac{1}{2} .
\end{aligned}
$$

The fact that $T_{V_{0}} \chi_{K}$ is continuous and $T_{V_{0}} \chi_{K}\left(x_{0}\right)>\frac{1}{2}$ implies that there is an open set $V\left(x_{0}\right)$ about $x_{0}$ such that $T_{V_{0}} \chi_{K}(x)>\frac{1}{2}$ for all $x \in V\left(x_{0}\right)$. By the choice of $x_{0}$, $V=V\left(x_{0}\right) \cap E \backslash K$ is a nonempty open set contained in $E \backslash K$ for which $x \in V$ implies $T_{V_{0}} \chi_{K}(x)>\frac{1}{2}$. It follows that $\frac{1}{2} \cdot \chi_{V} \leqq T_{V_{0}} \chi_{K} \cdot \chi_{E \backslash K}$. Since $V_{0} \subset U_{0}$, for any measurable set $A \subset V$ we have

$$
\begin{aligned}
\frac{1}{2} \cdot S_{B}\left(\chi_{A}\right) & \leqq S_{B}\left(\frac{1}{2} \chi_{V}\right) \leqq S_{B}\left(T_{V_{0}} \chi_{K} \cdot \chi_{E \mid K}\right) \\
& =S_{B}\left(\chi_{E \backslash K} \cdot\left(T_{V_{0}} \chi_{K}-\chi_{K}\right)\right) \leqq S_{B}\left(T_{V_{0}} \chi_{K}-\chi_{K}\right)<\varepsilon / 2
\end{aligned}
$$

Set $\delta=\frac{1}{2} \mu(V)>0$, and let $A$ be a measurable set with $\mu(A)<\delta$. Since $G$ has no atoms, there is a measurable set $A^{\prime}$ contained in $V$ satisfying $\mu\left(A^{\prime}\right)=\mu(A)$. The equimeasurability of $\chi_{A^{\prime}}$ with $\chi_{A}$ implies

$$
S_{B}\left(\chi_{A}\right)=S_{B}\left(\chi_{A^{\prime}}\right) \leqq S_{B}\left(\chi_{V}\right)<\varepsilon
$$

We now show that $C_{0}$ is dense in $\Lambda$. Let $f \in \Lambda$; we assume without loss of generality that $f \geqq 0$. Given $B \subset \Lambda^{*}$ normal, rearrangement invariant and weakly bounded, there is a compact 0 -neighborhood $U_{0}$ such that $S_{B}\left(T_{U_{0}} f-f\right)<\frac{1}{3}$. The function $\varphi=T_{U_{0}} f$ is nonnegative, uniformly continuous and contained in $\Lambda$. By the hypothesis of (2), there is an integer $n$ for which $S_{B}\left(\varphi-\varphi \chi_{K_{n}}\right)<\frac{1}{3}$. Setting $d=\sup _{x \in K_{n+1}} \varphi(x)$, we can find a $\delta>0$ such that $\mu(A)<\delta$ implies $S_{B}\left(\chi_{A}\right)<1 / 3 d$. Let $U$ be an open set such that $K_{n} \subset U \subset K_{n+1}$ and $\mu\left(U \mid K_{n}\right)<\delta$, and let $g$ be a continuous Urysohn function with its support contained in $U$ and having the properties that $g \equiv 1$ on $K_{n}$ and $g(x) \leqq 1$ for all $x \in G$. The function $g \cdot \varphi=\psi$ is a continuous function whose compact support is contained in $U$ satisfying

$$
\begin{aligned}
S_{B}\left(\varphi \cdot \chi_{K_{n}}-\psi\right) & =S_{B}\left(\varphi \cdot \chi_{K_{n}}-\varphi \cdot g\right)=S_{B}\left(\varphi \cdot\left(g-\chi_{K_{n}}\right)\right)=S_{B}\left(\varphi \cdot \chi_{U} \cdot\left(g-\chi_{K_{n}}\right)\right) \\
& =S_{B}\left(g \cdot \varphi \chi_{U \mid K_{n}}\right) \leqq d \cdot 1 \cdot S_{B}\left(\chi_{U \mid K_{n}}\right)<\frac{d}{3 d}=\frac{1}{3} .
\end{aligned}
$$


We finally have

$$
S_{B}(f-\psi) \leqq S_{B}(f-\varphi)+S_{B}\left(\varphi-\varphi \chi_{K_{n}}\right)+S_{B}\left(\varphi \chi_{K_{n}}-\psi\right)<1
$$

Thus $C_{0}$ is dense in $\Lambda$ for the strong topology. The Theorem is proved.

COROLLARY. If $\Lambda$ is a rearrangement invariant Köthe space over $G$ which contains all the constant functions, then $T_{U} f \rightarrow f(U \in \mathscr{U})$ strongly for each $f \in \Lambda$ if and only if the uniformly continuous functions are strongly dense in $\Lambda$.

Proof. To show sufficiency we observe that $S_{B}\left(\chi_{G}\right)<\infty$ for $B \subset \Lambda^{*}$ weakly bounded. From this we can show, as in the Theorem, that $T_{U} \varphi \rightarrow \varphi$ for any uniformly continuous $\varphi \in \Lambda$. We again use the denseness of the uniformly continuous functions and the fact that $S_{B}\left(T_{U} f\right) \leqq S_{B}(f)$ for seminorms $S_{B}$ generating the topology on $\Lambda$ to show $T_{U} f \rightarrow f(U \in \mathscr{U})$ strongly. The necessity part of the Corollary is obvious since $T_{U} f$ is uniformly continuous for $U$ compact.

REMARK. The $L^{p}$ spaces for $1 \leqq p<+\infty$ are spaces in which $T_{U} f \rightarrow f(U \in \mathscr{U})$. $L^{\infty}$ is a space which does not have this property; the continuous functions are not dense for the strong (norm) topology. We now give an example of a Köthe space that is not rearrangement invariant, in which the continuous functions of compact support are dense, and for which $f \in \Lambda$ implies $T_{U} f \in \Lambda$, but $T_{U} f$ does not converge to $f$ for the strong topology.

Let $G=R^{1}$ and let $\mu$ be Lebesgue measure on $R^{1}$. Construct sequences of positive numbers $\left\{a_{n}\right\}_{n=1}^{\infty},\left\{b_{n}\right\}_{n=1}^{\infty}$, and $\left\{c_{n}\right\}_{n=1}^{\infty}$ such that the following is true:

$$
a_{n}<b_{n}<c_{n}<a_{n+1} \ldots \text {; }
$$

$c_{n}-b_{n}=b_{n}-a_{n}=1 / n^{2}$ for each $n$;

if $A_{n}=\left(a_{n}, b_{n}\right)$ and $B_{n}=\left(b_{n}, c_{n}\right)(n=1,2, \ldots)$, then $\bigcup_{n=1}^{\infty} A_{n} \cup \bigcup_{n=1}^{\infty} B_{n}$ is contained in a compact interval.

Set $f=\sum_{n=1}^{\infty} n^{3 / 4} \cdot \chi_{A_{n}}$ and $g=\sum_{n=1}^{\infty} n^{3 / 4} \cdot \chi_{B_{n}}$. Both $f$ and $g$ are integrable, of compact support, and $f \cdot g \equiv 0$. Thus $f \in L_{g}^{1}$ which is Köthe space in which the continuous functions of compact support are dense, and in which $T^{h} f^{\prime} \in L^{1} g$ whenever $f^{\prime} \in L^{1} g$ (as $L^{1} g$ contains all the continuous functions). We show $\lim _{h \rightarrow 0} \int_{-\infty}^{\infty} T^{h} f(x) \cdot g(x) d x$ $=\infty$. Choosing $h$ sufficiently small, we will have

$$
\begin{aligned}
\int_{-\infty}^{\infty} T^{n} f(x) \cdot g(x) & d x=\sum_{n=1}^{\infty} \int_{B_{n}} \frac{1}{h} \cdot \int_{-h / 2}^{h / 2} f(x+t) \cdot g(x) d t d x \\
& \geqq \sum_{n=1}^{\infty} \frac{1}{h} \cdot \int_{-h / 2}^{0} \int_{B_{n}} n^{3 / 2} \cdot \chi_{A_{n}}(x+t) d x d t \\
& \geqq \sum_{n \leqq n(h)} \frac{1}{h} \int_{-h / 2}^{0} \int_{b_{n}}^{b_{n}+t} \chi_{A_{n}-t}(x) d x d t \quad(\text { where } n(h)=[\sqrt{ }(2 / h)]+1) \\
& =\sum_{n \leqq n(h)} n^{3 / 2} \cdot \frac{1}{h} \int_{-h / 2}^{0} t \cdot d t=\frac{h}{8} \sum_{n \leqq n(h)} n^{3 / 2} \geqq \frac{1}{10} \frac{1}{h^{1 / 4}} \rightarrow \infty \quad \text { as } h \rightarrow 0 .
\end{aligned}
$$




\section{REFERENCES}

1. J. Dieudonné, Sur les espaces de Köthe, J. Analyse Math. 1 (1951), 81-115. MR 12, 834.

2. S. Goes, Some compactness criteria for Köthe spaces, Dissertation, Northwestern University, Evanston, Ill., 1967 (unpublished).

3. G. Köthe and $O$. Toeplitz, Lineare Räume mit unendlichvielen Koordinaten und Ringe unendlicher Matrizen, J. Reine Angew. Math. 171 (1934), 193-226.

4. G. Köthe, Neubegründung der Theorie der vollkommenen Räume, Math. Nachr. 4 (1951), 70-80. MR 12, 615.

5. _- Die Stufenräume, eine einfache Klasse linearer vollkommener Räume, Math. Z. 51 (1948), 317-345. MR 10, 255.

6. G. Silverman, Strong topology on a rearrangement invariant Köthe space (unpublished).

7. —-, Rearrangement invariant Köthe spaces, Math. Ann. 189 (1970), 222-234.

Department of Mathematics, NorthWestern University, Evanston, Illinois 60201

Current address: 17-46 Country Club Road, Eatontown, New Jersey 07724 